TITLE:

\title{
Fgf20b is required for the ectomesenchymal fate establishment of cranial neural crest cells in zebrafish.
}

\section{$\operatorname{AUTHOR}(S):$}

Yamauchi, Hajime; Goto, Mami; Katayama, Mika; Miyake, Ayumi; Itoh, Nobuyuki

\section{CITATION:}

Yamauchi, Hajime ... [et al]. Fgf20b is required for the ectomesenchymal fate establishment of cranial neural crest cells in zebrafish.. Biochemical and Biophysical Research Communications 2011, 409(4): 705-710

\section{ISSUE DATE:}

2011-06-17

URL:

http://hdl.handle.net/2433/143594

\section{RIGHT:}

(C) 2011 Elsevier Inc.; This is not the published version. Please cite only the published version.; この論文は出版社版でありません。引用の際に は出版社版をご確認ご利用ください。 
Fgf20b is required for the ectomesenchymal fate establishment of cranial neural crest cells in zebrafish

Hajime Yamauchi ${ }^{1}$, Mami Goto ${ }^{1}$, Mika Katayama, Ayumi Miyake and Nobuyuki Itoh*

Department of Genetic Biochemistry, Kyoto University Graduate School of Pharmaceutical Sciences, Sakyo, Kyoto 606-8501, Japan

${ }^{1}$ These authors contributed equally to this work.

*Corresponding author:

Department of Genetic Biochemistry, Kyoto University Graduate School of Pharmaceutical Sciences, Yoshida-Shimoadachi, Sakyo, Kyoto 606-8501, Japan. Tel: +81-75-753-4540; Fax: +81-75-753-4600; E-mail address:

itohnobu@pharm.kyoto-u.ac.jp 


\begin{abstract}
In cranial skeletal development, the establishment of the ectomesenchymal lineage within the cranial neural crest is of great significance. Fgfs are polypeptide growth factors with diverse functions in development and metabolism. Fgf $20 \mathrm{~b}$ knockdown zebrafish embryos showed dysplastic neurocranial and pharyngeal cartilages. Ectomesenchymal cells from cranial neural crest cells were significantly decreased in $F g f 20 b$ knockdown embryos, but cranial neural crest cells with a non-ectomesnchymal fate were increased. However, the proliferation and apoptosis of cranial neural crest cells were essentially unchanged. Fgfrl knockdown embryos also showed dysplastic neurocranial and pharyngeal cartilages. The present findings indicate that Fgf20b is required for ectomesenchymal fate establishment via the activation of Fgfrl in zebrafish.
\end{abstract}

Key words: Fgf; Fgf20b; cranial neural crest; pharyngeal arch; ectomesenchyme; zebrafish 


\section{Introduction}

In vertebrate embryonic development, the formation of a head skeleton comprising the cranium and mandible is essential for the establishment of a proper physical frame. Most of the bones and cartilage in the head skeleton are derived from cranial neural crest cells [1]. After developing at the neural plate and epidermis, neural crest cells emigrate from the dorsal neural tube to their final destination during embryonic development. Cranial neural crest cells differentiate into ectomesenchyme and non-ectomesenchyme. In cranial skeletal development, the ectomesenchyme terminally differentiates into bones, cartilages, and associated connective tissues and generates much of the craniofacial skeleton. The establishment of the ectomesenchymal lineage within the cranial neural crest is of great significance [2].

Fgfs are polypeptide growth factors with diverse functions in development and metabolism. The Fgf family comprises 22 and 28 members in humans/mice and zebrafish, respectively $[3,4]$. We originally identified Fgf20 as a neurotrophic factor that acts via the activation of Fgf receptor 1 (Fgfr1) in a paracrine manner [5, 6]. Fgf20 is expressed in mouse embryonic calvaria and developing limbs [7]. In addition, Fgf20 is potentially required for sensory cell specification in mouse cochlear development [8]. Fgf20 also regulates the development of embryonic and neural stem cells into tyrosine hydroxylase-positive neurons in cultured cells $[9,10]$. However, the physiological roles of Fgf20 remain unclear.

Zebrafish Fgf20 is an ortholog of human/mouse Fgf20. Zebrafish Fgf20a and Fgf20b are paralogs $[3,11]$. Fgf20a is required for fin regeneration, the restriction of neurogenesis, and the segment center progenitor population $[11,12]$. However, the roles of Fgf20b remain unclear. In this paper, we report that Fgf20b is required for the ectomesenchymal fate establishment of cranial neural crest cells in zebrafish. 


\section{Materials and methods}

\subsection{Fish maintenance}

Zebrafish (Danio rerio) were maintained and the developmental stages of embryos were determined as described [13].

\subsection{Whole mount in situ hybridization and staining}

Whole mount in situ hybridization was performed as described [13]. The Fgf2Ob probe was synthesized using a full-length cDNA-containing plasmid. Other probes used were zebrafish foxd3 [14], sox10 [15], dlx2 [16], col2ala [17] , and Fgfrl [13]. Cartilage was stained with Alcian blue as described [18].

\subsection{Morpholino injection}

Antisense MOs were synthesized by Gene-Tools, LLC (Corvallis, OR). The sequences were as follows: splice-site-targeted Fgf20b MO1, 5'-AAGACAAGTCTGCTTACTGACCAT-3'; splice-site-targeted Fgf20b MO2, 5'-TTCCAAAATACCTGGAGAAGAATAA-3'. A splice-site-targeted Fgfrl MO was used as described [13]. The Fgf20b MO1 (20 or $30 \mathrm{ng}$ ), Fgf20b MO2 (4 or 6 ng), and Fgfrl MO (12 ng) were injected into one- to two-cell embryos. To examine each MO's efficacy, RNA was isolated from wild-type, $F g f 20 b$ MO1-injected or $F g f 20 b$ MO2-injected embryos. cDNA was amplified from the RNA by RT-PCR. 


\subsection{Rescue experiments}

Capped zebrafish $F g f 20 b$ sense mRNA was prepared with the mMESSAGE mMACHINE kit (Ambion) according to the manufacturer's directions from linearized $p C S 2+$ plasmids containing zebrafish $F g f 20 b$ cDNA. To perform rescue experiments, $0.05 \mathrm{pg}$ of $F g f 20 b$ sense mRNA was injected separately, immediately after the injection of Fgf20b MO1 (20 ng) into two-cell embryos.

\subsection{Detection of proliferating and apoptotic cells}

Proliferating cells were detected by immunohistochemistry using an anti-phosphorylated H3 (H3P) antibody (Upstate Biotechnology). Apoptotic cells were examined by TUNEL assay using the DeadEnd colorimetric detection kit (Promega) as described.

\section{Results and discussion}

\subsection{Spatiotemporal expression pattern of Fgf20b}

We examined the spatiotemporal expression pattern of $F g f 20 b$ in zebrafish embryos by RT-PCR (Fig. 1A) and whole mount in situ hybridization (Fig. 1B-G). Fgf $20 b$ expression was barely detectable at 12 hpf. However, the expression increased gradually until at least $120 \mathrm{hpf}$. This expression pattern is similar to that of Fgf2Oa. At 14 hpf, Fgf2Ob was expressed in the region where migrating cranial neural crest cell subpopulations possibly exist. Fgf2Ob was expressed in the pharyngeal arch at $20 \mathrm{hpf}$ and further in the mandibular arch (the first pharyngeal arch), hyoid arch (the second pharyngeal arch), gill arches, and the otic vesicle at 36 
hpf. The pattern for $F g f 20 b$ is quite distinct from that for $F g f 20 a$, which is mainly expressed in the hindbrain at $16 \mathrm{hpf}$ and a cluster of cells in each rhombomere after $18 \mathrm{hpf}$ [12]. Cranial neural crest cells arise from dorsal and lateral regions of the neural ectoderm, and then migrate to the pharyngeal arches, where they form neural and skeletal tissues [18]. The expression of $F g f 20 b$ suggests potential roles in the development of cranial neural crest cells.

\subsection{Inhibition of Fgf20b functions results in mandibular hypoplasia}

To inhibit $F g f 20 b$ functions in zebrafish embryos, we used splice-site-targeted $F g f 20 b$ MOs (Fgf20b MO1 and MO2) (Fig. S1A). RNA was isolated from wild-type, Fgf20b MO1-injected, or Fgf2Ob MO2-injected embryos at $48 \mathrm{hpf}$. Fgf20b cDNA was amplified from the RNA by RT-PCR using the P1 and P2 primers (Fig. S1B). Mature Fgf20b mRNA levels decreased significantly in the $F g f 20 b$ $\mathrm{MO} 1$ and $\mathrm{Fgf2Ob} \mathrm{MO} 2$-injected embryos in a dose-dependent manner. In addition, mis-spliced Fgf2Ob mRNA forms were significantly detected. These results indicate that $F g f 20 b \mathrm{MO} 1$ and $\mathrm{MO} 2$ significantly impaired the maturation of $F g f 20 b$ mRNA. We analyzed $F g f 20 b$ knockdown embryos at 5 days post fertilization (dpf) (Fig. 2A-H). The Fgf20b MO1-injected embryos showed impaired head regions ( $75.6 \%, \mathrm{n}=45$ by $20 \mathrm{ng} ; 83.3 \%, \mathrm{n}=24$ by $30 \mathrm{ng}$ ). In particular, mandibular hypoplasia, a significant reduction in the size of the lower jaw, was observed. The $F g f 20 b$ MO2-injected embryos showed similar phenotypes to the $F g f 20 b$ MO1-injected embryos $(86.1 \%, n=36$ by $4 n g ; 98.7 \%, n=44$ by $6 n g)$. However, the mandibular hypoplasia in Fgf20b MO1(20 ng)-injected embryos was significantly rescued by the injection of capped $F g f 20 b$ mRNA $(69.2 \%$, n=26 by 0.025 pg mRNA; $83.3 \%$, n=24 by 0.05 pg mRNA). These results suggest that $F g f 20 b$ is required for the proper formation of the lower jaw. 


\subsection{Fgf20b is required for neurocranial and pharyngeal cartilage to form}

We examined $F g f 20 b$ knockdown embryos at 5 dpf by histological analysis with Alcian blue, which stained sulfated glycosaminoglycan-rich tissues such as cartilage (Fig. 2I-N). The size of the anterior neurocranial cartilage, which includes the ethmoid plate and trabeculae, and pharyngeal cartilage was significantly reduced in Fgf20b knockdown embryos $(75.6 \%, \mathrm{n}=45$ by $20 \mathrm{ng}$ MO1; MO2: $86.1 \%, \mathrm{n}=36$ by 4 ng MO2). In particular, the first and second pharyngeal cartilages such as Meckel's cartilage, palatoquadrates, basihyal and ceratohyals were dysplastic. These results indicate that Fgf20b is required for the proper formation of neurocranial and pharyngeal cartilages.

3.4. Fgf20b is required for the ectomesenchymmal fate establishment of cranial neural crest cells

Cranial neural crest cells have the potential to generate mesenchymal derivatives, which can be classified into two mesenchymes, ectomesenchyme and non-ectomesenchyme [19]. In zebrafish, these cells are spatially segregated in the premigratory neural crest cells [15]. After migrating to pharyngeal arches, ectomesenchymal cells form cartilage and connective tissues [2]. The drosophila distal-less homeobox gene $d l x 2$ is a marker for ectomesenchymal cells [2]. $d l x 2$ expression was significantly decreased in $F g f 20 b$ knockdown embryos (20 ng MO1) (78.8\%, n=33) (Fig. 3A, B). The forkhead box-containing transcription factor gene foxd3 is expressed in premigratory and early migratory neural crest cells [20]. The HMG box-containing transcription factor gene sox10 is expressed in the migratory neural crest cells [2]. foxd3 and sox10 are markers for neural crest cells with a 
non-ectomesenchymal fate [2]. foxd3 and sox10 expression was significantly increased in $F g f 20 b$ knockdown embryos $(20 \mathrm{ng}$ MO1) at $24 \mathrm{hpf}(67.7 \%$, n=31 and $77.4 \%, \mathrm{n}=32$, respectively) (Fig. 3C-F). These results indicate that the neural crest cells did not properly differentiate into ectomesenchymal cells in Fgf2Ob knockdown embryos

3.5. Fgf20b is not required for the endoderm to form in neurocaranial and pharyngeal cartilages

We examined the roles of Fgf20b after the migration of cranial neural crest cells to the pharyngeal arches. The type II collagen gene col2ala is a marker for differentiating chondrocytes [17]. col2ala expression was also decreased in the pharyngeal arches of Fgf20b knockdown embryos (90.6 \%, n=32) (Fig. 3G, H). These results indicate that a decrease in ectomesenchymal cells resulted in diminished chondrogenesis.

The pharyngeal endoderm interacts with the neural crest during the formation of neurocranial and pharyngeal cartilages [21]. The NK2 transcription factor gene $n k x 2.3$ is a marker for the pharyngeal endoderm [22]. $n k x 2.3$ expression in the pharyngeal endoderm was essentially unchanged in $F g f 20 b$ knockdown embryos (20 ng MO1) at $36 \mathrm{hpf}(87.5 \%, \mathrm{n}=32)$ (Fig. 3I, J). The bHLH transcription factor gene hand2, which is expressed in the ventral endoderm during the pharyngeal arch's development, is required for the formation of the ventral pharyngeal cartilage including the lower jaw [23]. hand2 expression was also essentially unchanged in the Fgf2Ob knockdown embryos (20 ng MO1) at $24 \mathrm{hpf}(77.4 \%, \mathrm{n}=31)$ (Fig. 3K, L). These results suggest that Fgf20b is not essential to the formation of the endoderm in the pharyngeal cartilage. 
3.6. Fgf20b is not critically involved in the proliferation and survival of cranial neural crest cells

In $F g f 20 b$ knockdown embryos, the number of cranial neural crest cells with a non-ectomesenchymal fate was increased, whereas the number of ectomesenchymal cells was decreased. Therefore, we examined the proliferation and survival of cranial neural crest cells in Fgf2Ob knockdown embryos. Proliferating cell levels examined by immunohistochemistry with phosphohistone-H3 antibody were essentially unchanged in Fgf20b knockdown embryos (20 ng MO1) at 14 and $24 \mathrm{hpf}$ (Fig. 4A-H, Q), indicating that the proliferation of cranial neural crest cells was essentially unchanged in Fgf20b knockdown embryos. Apoptotic cell levels examined by the TUNEL assay were only slightly increased in Fgf20b knockdown embryos (Fig. 4I-P, R), indicating that the survival of cranial neural crest cells was not critically affected in $F g f 20 b$ knockdown embryos.

\subsection{Fgfrl is required for neurocranial and pharyngeal cartilage to form}

Fgf20 functions via the activation of Fgfrl in a paracrine manner [6]. Fgf20b is also expected to function via the activation of Fgfrs in a paracrine manner. The zebrafish Fgfr gene family comprises four members, Fgfrl-Fgfr4 [13]. We generated Fgfrl-Fgfr4 knockdown embryos injected with splice-site targeted Fgfrl-Fgfr4 MOs as described previously [13]. The knockdown embryos at $5 \mathrm{dpf}$ were examined by histological analysis with Alcian blue staining. Aberrant neurocranial and pharyngeal cartilages were observed only in Fgfrl knockdown embryos ( $70 \%, \mathrm{n}=10$ by $12 \mathrm{ng}$ ) (Fig. S2A-D). Fgfrl was expressed in the region where migrating cranial neural crest cell subpopulations possibly exist at $14 \mathrm{hpf}$ (Fig. S2E). Later, Fgfrl was expressed in the pharyngeal arches at $24 \mathrm{hpf}$ (Fig. S2F). 
This expression pattern is essentially consistent with that reported [24]. In addition, Fgf20 potentially binds to and activates Fgfr1 [6]. These results indicate that Fgf20b possibly functions via the activation of Fgfr1

Zebrafish Fgf20a functions in fin regeneration, the restriction of neurogenesis, and the segment center progenitor population $[11,12]$. The present results indicate that Fgf20b has roles distinct from those of Fgf20a. In conclusion, Fgf20b contributes to head skeletal development by establishing the ectomesenchymal fate of cranial neural crest cells in zebrafish.

\section{Acknowledgements}

This work was supported by a Grant-in-aid for Scientific Research from the Ministry of Education, Science, Culture and Sports of Japan and the Takeda Science Foundation, Japan.

\section{References}

[1] J.A. Helms, D. Cordero, M.D. Tapadia, New insights into craniofacial morphogenesis. Development 132 (2005) 851-861.

[2] A. Blentic, P Tandon, S. Payton, J. Walshe, T. Carney, R.N. Kelsh, I. Mason, A. Graham, The emergence of ectomesenchyme. Dev. Dyn. 237 (2008) 592-601.

[3] N. Itoh, M. Konishi, The zebrafish fgf family. Zebrafish 4 (2007) 179-186.

[4] N. Itoh, D.M. Ornitz, Fibroblast growth factors: from molecular evolution to roles in development, metabolism and disease. J. Biochem. 149 (2011) 121-130. 
[5] S. Ohmachi, Y. Watanabe, T. Mikami, N. Kusu, T. Ibi, A. Akaike, N. Itoh, FGF-20, a novel neurotrophic factor, preferentially expressed in the substantia nigra pars compacta of rat brain. Biochem. Biophys. Res. Commun. 277 (2000) 355-360.

[6] S. Ohmachi, T. Mikami, M. Konishi, A. Miyake, N. Itoh, Preferential neurotrophic activity of fibroblast growth factor-20 for dopaminergic neurons through fibroblast growth factor receptor-1c. J. Neurosci. Res. 72 (2003) 436-443.

[7] M.K. Hajihosseini, J.K. Heath, Expression patterns of fibroblast growth factors-18 and -20 in mouse embryos is suggestive of novel roles in calvarial and limb development. Mech. Dev. 113 (2002) 79-83.

[8] T. Hayashi, C.A. Ray, O. Bermingham-McDonogh, Fgf20 is required for sensory epithelial specification in the developing cochlea. J. Neurosci. 28 (2008) 5991-5999.

[9] C. Grothe, M. Timmer, T. Scholz, C. Winkler, G. Nikkhah, P. Claus, N. Itoh, E. Arenas, Fibroblast growth factor-20 promotes the differentiation of Nurrl-overexpressing neural stem cells into tyrosine hydroxylase-positive neurons. Neurobiol. Dis. 17 (2004) 163-170.

[10] Y. Takagi, J. Takahashi, H. Saiki, A. Morizane, T. Hayashi, Y. Kishi, H. Fukuda, Y. Okamoto, M. Koyanagi, M. Ideguchi, H. Hayashi, T. Imazato, H. Kawasaki, H. Suemori, S. Ohmachi, H. Iida, N. Itoh, N. Nakatsuji, Y. Sasai, N. Hashimoto, Dopaminergic neurons generated from monkey embryonic stem cells function in a Parkinson primate model. J. Clin. Invest. 115 (2005) 102-109.

[11] G.G. Whitehead, S. Makino, C.L. Lien, M.T. Keating, fgf20 is essential for initiating zebrafish fin regeneration. Science 310 (2005) 1957-1960. 
[12] R. Gonzalez-Quevedo, Y. Lee, K.D. Poss, D.G. Wilkinson, Neuronal regulation of the spatial patterning of neurogenesis. Dev. Cell 18 (2010) 136-147.

[13] Y. Nakayama, A. Miyake, Y. Nakagawa, T. Mido, M. Yoshikawa, M. Konishi, N. Itoh, Fgf19 is required for zebrafish lens and retina development. Dev. Biol. 313 (2008) 752-766.

[14] J. Odenthal, C. Nüsslein-Volhard, fork head domain genes in zebrafish. Dev. Genes Evol. 208 (1998) 245-258.

[15] K.A. Dutton, A. Pauliny, S.S. Lopes, S. Elworthy, T.J. Carney, J. Rauch, R. Geisler, P. Haffter, R.N. Kelsh, Zebrafish colourless encodes sox 10 and specifies non-ectomesenchymal neural crest fates. Development 128 (2001) 4113-4125.

[16] M.A. Akimenko, M. Ekker, J. Wegner, W. Lin, M. Westerfield, Combinatorial expression of three zebrafish genes related to distal-less: part of a homeobox gene code for the head. J. Neurosci. 14 (1994) 3475-3486.

[17] Y.L. Yan, K. Hatta, B. Riggleman, J.H. Postlethwait, Expression of a type II collagen gene in the zebrafish embryonic axis. Dev. Dyn. 203 (1995) 363-376.

[18] T.F. Schilling, T. Piotrowski, H. Grandel, M. Brand, C.P. Heisenberg, Y.J. Jiang, D. Beuchle, M. Hammerschmidt, D.A. Kane, M.C. Mullins, F.J. van Eeden, R.N. Kelsh, M. Furutani-Seiki, M. Granato, P. Haffter, J. Odenthal, R.M. Warga, T. Trowe, C. Nüsslein-Volhard, Jaw and branchial arch mutants in zebrafish I: branchial arches. Development 123 (1996) 329-344.

[19] N.M. Le Douarin, E. Dupin, Multipotentiality of the neural crest. Curr. Opin. Genet. Dev. 13 (2003) 529-536.

[20] R.N. Kelsh, J.S. Eisen, The zebrafish colourless gene regulates development of non-ectomesenchymal neural crest derivatives. Development 127 (2000) 
515-525.

[21] N.B. David, L. Saint-Etienne, M. Tsang, T.F. Schilling, F.M. Rosa, Requirement for endoderm and FGF3 in ventral head skeleton formation. Development 129 (2002) 4457-4468.

[22] K.H. Lee, Q. Xu, R.E. Breitbart, A new tinman-related gene, nkx2.7, anticipates the expression of nkx2.5 and nkx2.3 in zebrafish heart and pharyngeal endoderm. Dev. Biol. 180 (1996) 722-731.

[23] C.T. Miller, D. Yelon, D.Y. Stainier, C.B. Kimmel, Two endothelin 1 effectors, hand2 and bapx1, pattern ventral pharyngeal cartilage and the jaw joint. Development 130 (2003) 1353-1365.

[24] N. Rohner, M. Bercsényi, L. Orbán, M.E. Kolanczyk, D. Linke, M. Brand, C. Nüsslein-Volhard, M.P. Harris, Duplication of fgfr1 permits Fgf signaling to serve as a target for selection during domestication. Curr. Biol. 19 (2009) 1642-1647.

\section{Figure legends}

Fig. 1. Spatiotemporal expression pattern of $F g f 20 a$ and $F g f 20 b$. (A) The expression of Fgf2Oa, Fgf2Ob, and efl $\alpha$ as a control in embryos was examined by RT-PCR. (B-G) The expression of $F g f 20 b$ in embryos was examined by whole mount in situ hybridization. Arrows at 14 and $20 \mathrm{hpf}$ indicate possible regions of migrating cranial neural crest cell subpopulations and the pharyngeal arch, respectively. pa1, the first pharyngeal arch (the mandibular arch); pa2, the second pharyngeal arch (the hyoid arch); ga, gill arches; ov, the otic vesicle. Lateral views (B, D, F), dorsal views $(\mathrm{C}, \mathrm{E}, \mathrm{G})$. 
Fig. 2. The effect of Fgf20b knockdown on morphogenesis. (A-F) Two-cell embryos injected with $\mathrm{Fg} f 20 \mathrm{BO} \mathrm{MO}$ and $\mathrm{Fg} f 20 b \mathrm{MO} 2$ at 5 dpf. In contrast to wild-type embryos, there was a significant reduction in the size of the lower jaw in Fgf20b MO1- or MO2-injected embryos. Arrows indicate the aberrant lower jaw. $(\mathrm{G}, \mathrm{H})$ The reduction in the size of the lower jaw in Fgf20b MO1 embryos was clearly prevented by the injection of $F g f 20 b$ mRNA. Lateral views of the embryos are shown. The head region in $\mathrm{A}, \mathrm{C}, \mathrm{E}$ and $\mathrm{G}$ is shown at a higher magnification in B, D, F and H, respectively. (I-N) The effect of $F g f 20 b$ knockdown on neurocranial and pharyngeal cartilages. Alcian blue stained cartilage elements at $5 \mathrm{dpf}$ in wild-type embryos, Fgf2Ob MO1-injected, and Fgf2Ob MO2-injected embryos. In Fgf20b knockdown embryos, the size of ethmoid plate, trabeculae and pharyngeal cartilage were significantly reduced in size. Arrows indicate the anterior neurocranial cartilage including ethmoid plate, trabeculae and pharyngeal cartilage. bh, basihyal; ch, ceratohyal; e, ethmoid plate; m, Meckel's cartilage; pq, palatoquadrate; t, trabeculae. Lateral views (I, K, M); ventral views (J, L, N).

Fig. 3. The effect of $F g f 20 b$ knockdown on the commitment of cranial neural crest cells (A-F). (A, B) The expression of $d l x 2$ in embryos at 24 hpf. Arrows indicate the expression of $d l x 2$ in ectomesencymal cells. The expression of $d l x 2$ was significantly decreased in $F g f 20 b$ knockdown embryos. (C-F) The expression of foxd 3 and sox 10 in embryos at $24 \mathrm{hpf}$. Arrows indicate the expression of foxd 3 and sox10 in cranial neural crest cells with a non-ectomesenchymal fate. The expression of both foxd3 and sox10 was significantly increased in Fgf $20 \mathrm{~b}$ knockdown embryos.sox10 was also expressed in the otic vesicle (ov). Dorsal views of embryos are shown. Chondrogenesis and endoderm formation neurocranial and pharyngeal arches in Fgf20b knockdown embryos (G-L). (G, H) The expression of col2ala, which is required for chondrogenesis, in embryos. 
Arrows indicate the expression of col2ala in the pharyngeal arches. The expression of col2ala was significantly decreased in Fgf2Ob knockdown embryos. (I-L) The expression of $n k x 2.3$ and hand2, markers for the pharyngeal endoderm, in embryos. The expression of $n k x 2.3$ and hand2 was essentially unchanged in Fgf2Ob knockdown embryos. Lateral views (G, H, K, L); dorsal views (I, J).

Fig. 4. Proliferation and survival of cranial neural crest cells in Fgf2Ob knockdown embryos. (A-H) Proliferating cells, phospho-histone H3-positive cells, were examined. The regions between arrowheads indicate areas of migratory cranial neural crest cells (A, B) and pharyngeal arches (C, D). Arrows indicate phospho-histone H3-positive cells in the sections (E-H). (Q) The H3P-positive cells in the regions were examined quantitatively. (I-P) Apoptotic cell levels were examined by TUNEL assay. The regions between arrowheads indicate areas of migratory cranial neural crest cells $(\mathrm{I}, \mathrm{J})$ and pharyngeal arches $(\mathrm{K}, \mathrm{L})$. Arrows indicate apoptotic cells. (R) The apoptotic cells in the regions were examined quantitatively. Lateral views (A-D, I-L); sections of embryos (E-H, M-P). Error bars correspond to the standard deviation of the mean. Asterisk; $P<0.01$ by Student's $t$ test. The results are the mean \pm S.D. for 3 independent sections from 5 embryos 


\section{Fig.1}

A

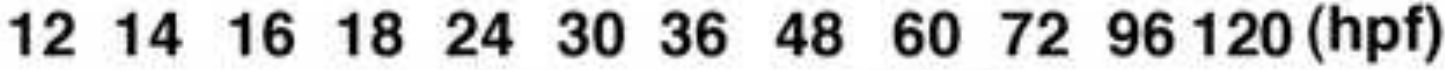

Fgf20a

Fgf20b

ef1 $\alpha$

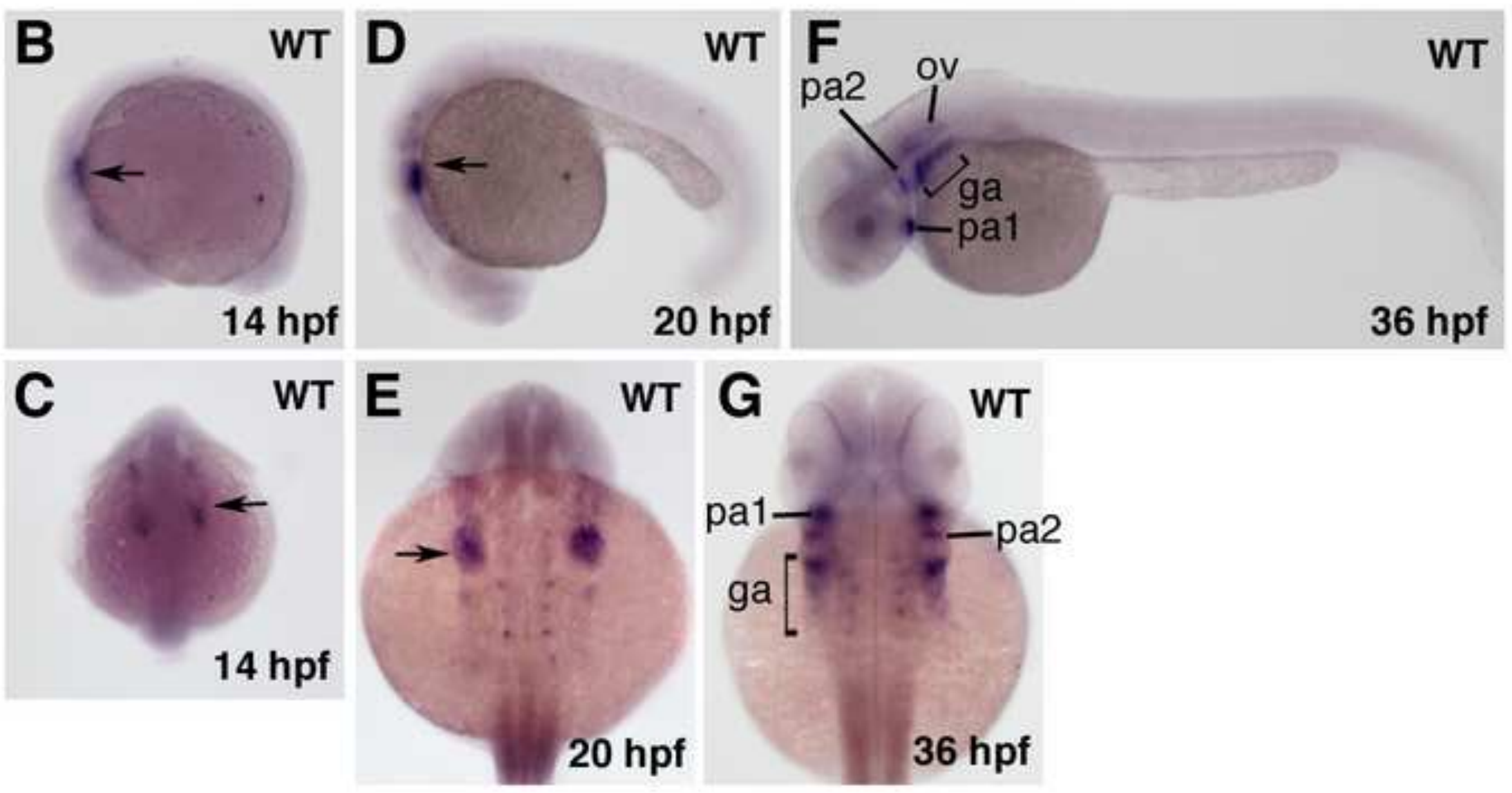


F: 京都大学

C 슬.

\section{Fig.2}

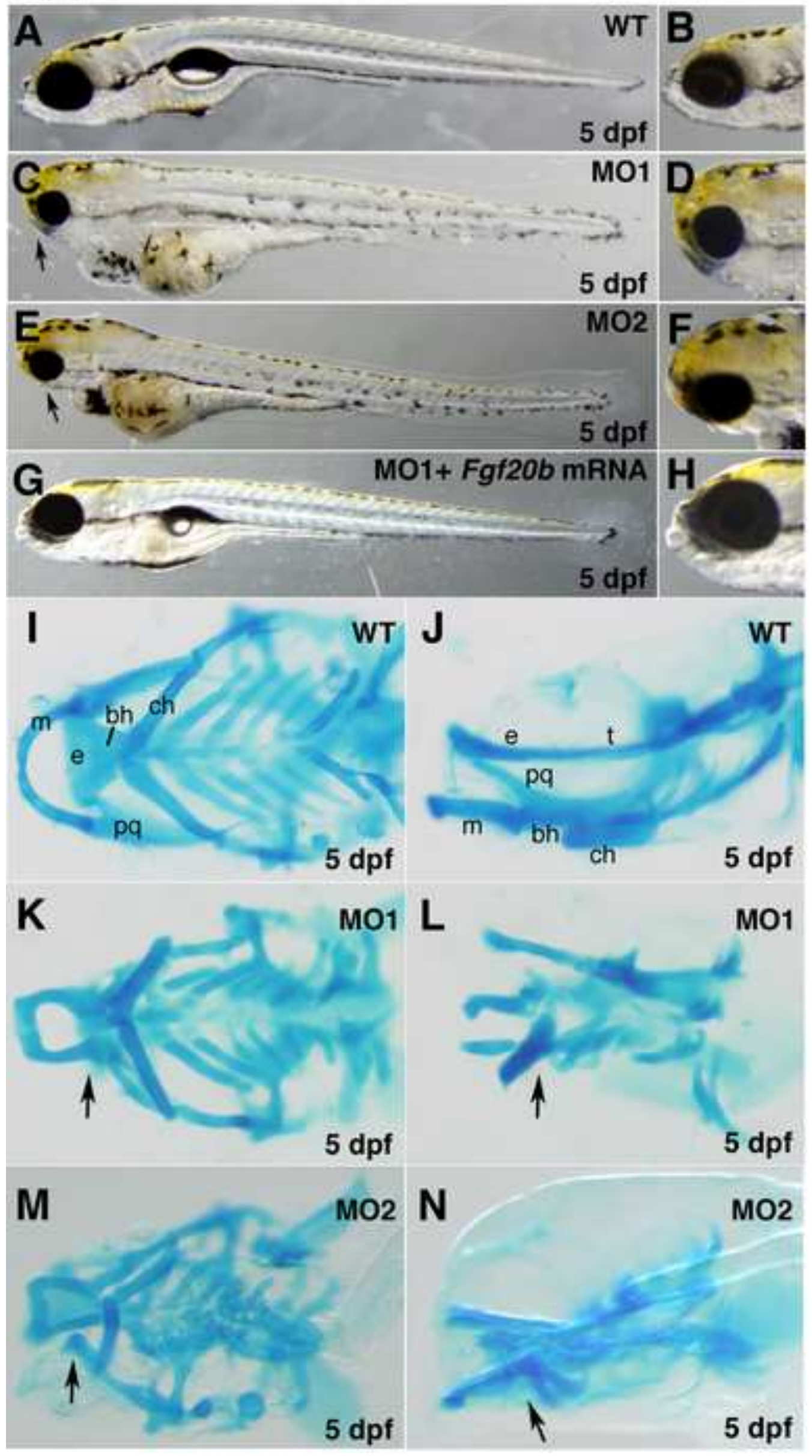


Fig.3

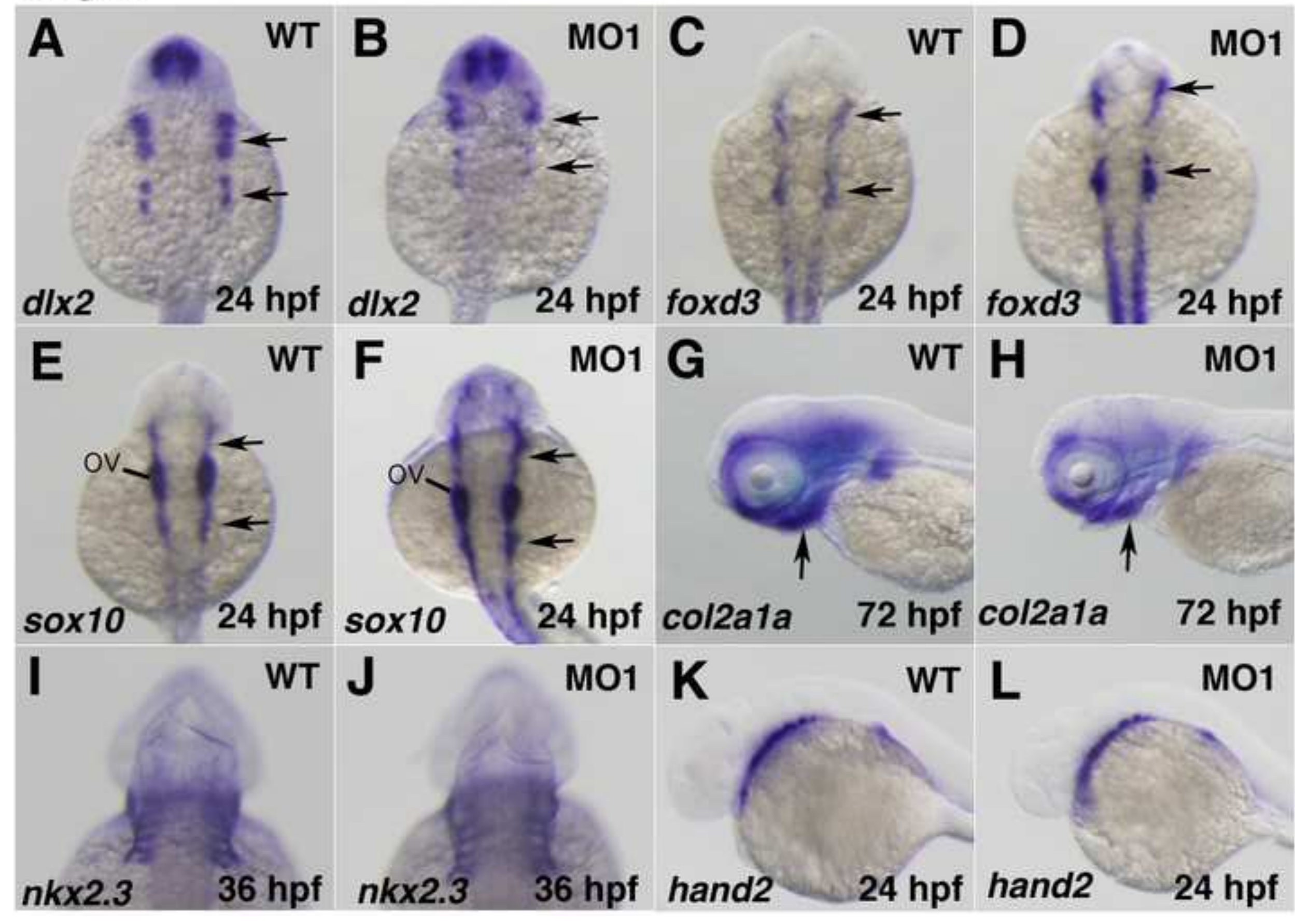

$n k \times 2.3$ $36 \mathrm{hpf} n k \times 2.3$ 


\section{Fig.4}

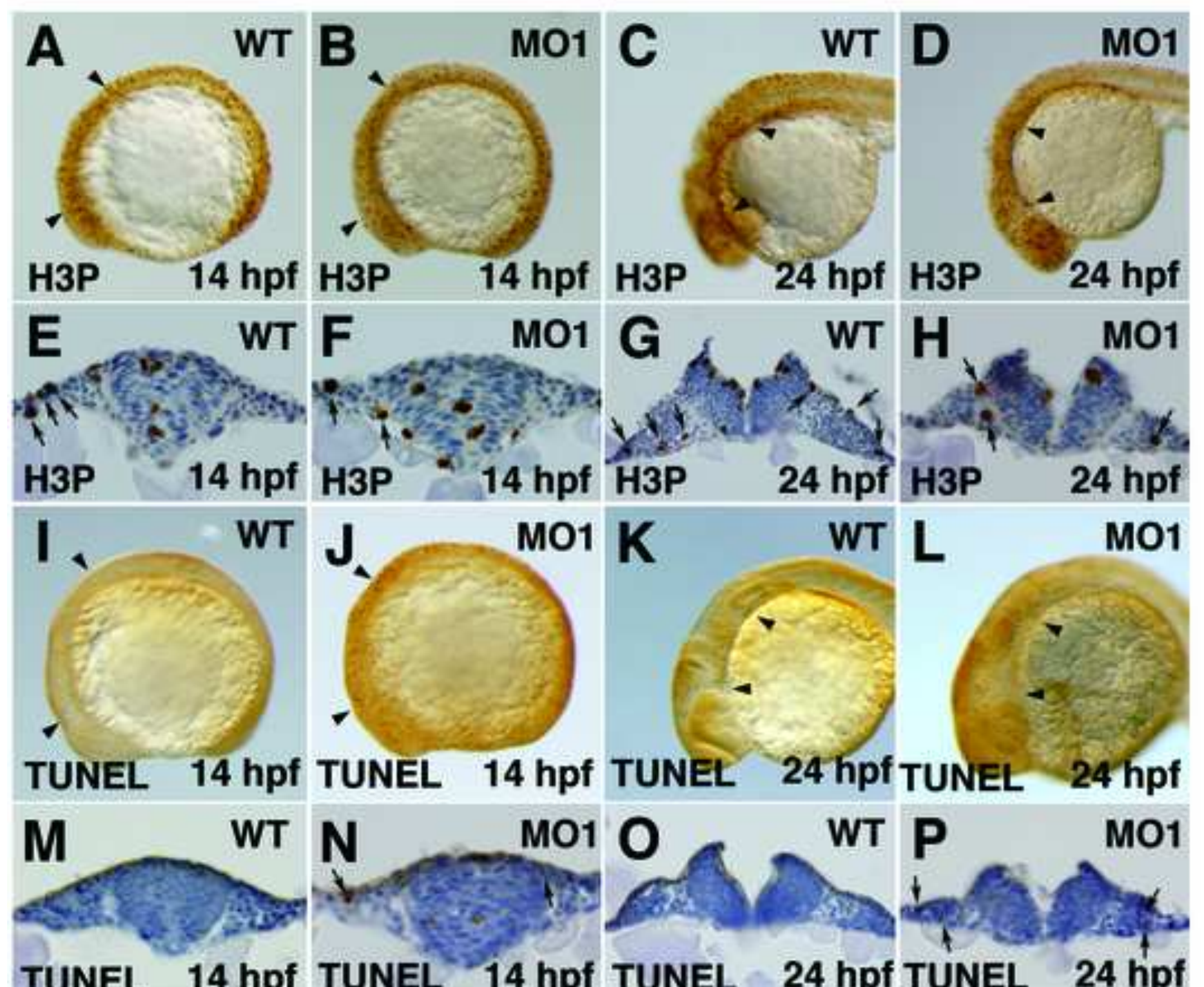

TUNEL $14 \mathrm{hpf}$ TUNEL $14 \mathrm{hpf}$ TUNel $24 \mathrm{hpf}$ TUNEL $24 \mathrm{hpf}$
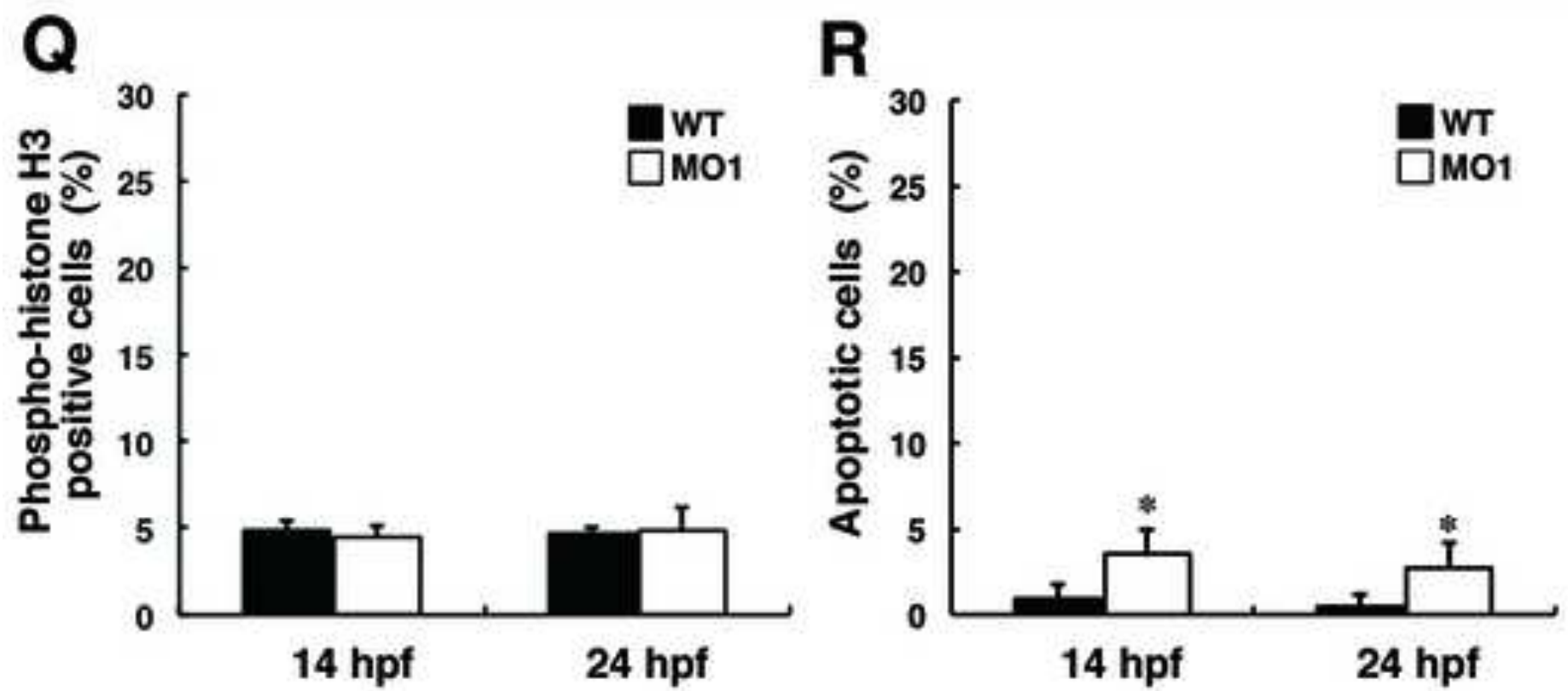